\title{
Subway operation safety evaluation based on IAHP-entropy weight method
}

\author{
J.X.Zhao ${ }^{\mathrm{a}}$, D.D.Wu ${ }^{\mathrm{b}}$ and M.M.Wang ${ }^{\mathrm{c}}$ \\ School of Management, Qingdao Technological University, Qingdao, China \\ azhjxqdsd@163.com, b1170331391@qq.com, lunwenzhyyx@126.com
}

\begin{abstract}
Keywords: IAHP; Game theory; Combination weighting method; Subway operation.
Abstract. Aiming at the subway operation safety assessment of empowerment as relatively one-sided, the game theory method is adopted to combine the improved AHP and the entropy weight method. On the basis of considering the factors of passenger flow, a system of evaluation index of metro operation safety is constructed.Taking Qingdao Metro Line 3 as an example, the weight of each index is calculated by using this model, and the influence degree of each indicator on the safety of subway operation is judged, and the comprehensive evaluation of Qingdao metro line 3 is carried out by combining the principle of fuzzy mathematics.
\end{abstract}

\section{Introduction}

As a relatively closed operation body, the subway system has a complex operation system. Once an accident occurs, it not only causes huge economic losses, but also threatens the safety of people's lives. Therefore, operation safety is the premise of realizing efficient operation of the subway system, and evaluation of the safety of subway operating system can effectively ensure the operation's safety and reliability.

In safety evaluation, evaluation model construction and index empowerment are the main problems to be considered. At present,there are three methods like subjective weighting method and the objective weighting method and combination weighting method.The subjective weighting method is based on the relative importance of expert knowledge and experience to determine the index, which is used to calculate the index weight, mainly in AHP, network analysis, such as Jia reservoir et al. [1] introduced the AHP to the safety evaluation of metro operation system, and calculated the main factors that influence subway operation safety.Objective method is based on the statistical results of raw data to determine the weights, the evaluation results with strong mathematical theory, mainly include the entropy weight method, grey correlation analysis method, etc., such as Jiang Ting [2] combined with the entropy theory to evaluate the risk of subway operation system. The combination of weighting method is a comprehensive consideration of subjective and objective factors to determine the weight and overcome the defects of the single method of empowerment.Commonly used a combination of empowerment method with linear weighted method, multiplicative synthesis method, etc., such as Wang [3] by using analytic hierarchy process and entropy value method combination of empowerment method to evaluate the indicators affecting the safety of urban rail transit lines operating.

Although the above-mentioned combination of weighting method overcomes the defect of single weight method, it lacks validity in determining the weighted coefficient, which affects the rationality of weight. Game theory studies two main and two or more factors in confrontational or competitive situation, how to make good decisions for scientific questions, the empowerment for subway operation safety evaluation index combination provides a new train of thought, based on this, this paper use the game theory model build up on the improved analytic hierarchy process and entropy weight method combination, the combined method is proposed based on game theory, in order to the subway operation safety assessment make reasonable, accurate and scientific evaluation. 


\section{Subway operation safety evaluation index system}

The change of subway passengers seriously affects the safety of subway operation system, to bring stability to the great subway operation system. Based on this, this paper considers the influence factors on the safety of passenger train operation in constructing the index system,combined with the safety evaluation standard of metro operation [9] and literature [8], in the end, the system of subway operation safety evaluation index system including passenger flow factor, equipment factor, management factor and environmental factor is established, as shown in figure 1.

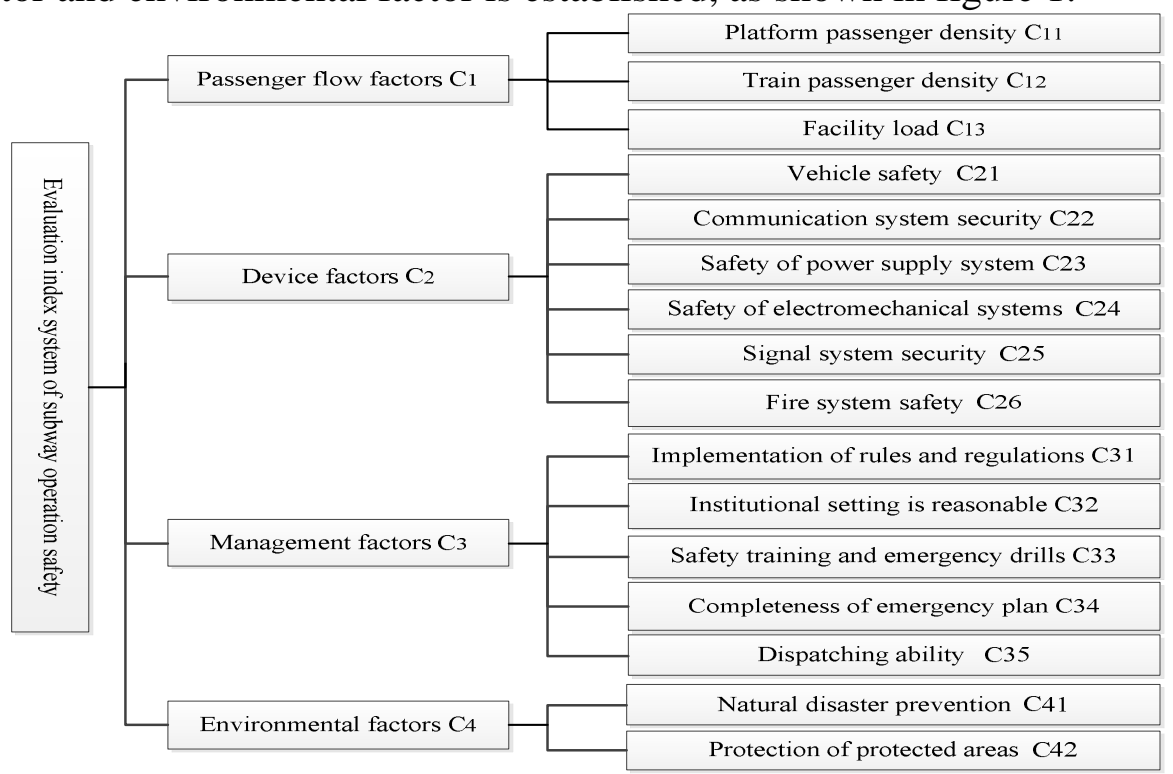

Fig. 1 The Index System of subway operation safety evaluation

\section{Determine portfolio weights based on game theory}

This paper determined the subway operation safety evaluation index weight of idea is: first of all, based on improved analytic hierarchy process (IAHP) the subjective weights are calculated, and then entropy value method is used to determine the objective weight, finally according to game theory integration means to calculate the combination weights.

\section{IAHP determines subjective weights}

The traditional analytical method using the nine scale method to generate the judgment matrix, and IAHP is the combination of the three scale method to generate interpretation matrix for experts to make relatively important decisions in 22 factors, eliminating the need for the judgment matrix consistency test link, improve computing efficiency and meet the requirements of accuracy improvement. The general steps of hierarchy the analysis method for [5]:

Determine the comparison matrix.

Using the three scale method to the same level of importance of different index for 22, compared to establish evaluation index matrix $\mathrm{K}$, which said i factors with respect to the degree of important factors for $\mathrm{j}$, and meet $k_{i i}$,

$k_{i j}=\left\{\begin{array}{c}0, \text { Factori is not important than factor } \mathrm{j} \\ 1, \text { Factori is as importantas factor } \mathrm{j} \\ 2, \text { Factor I is moreimportant than factor } \mathrm{j}\end{array}\right.$

Construction judgment matrix. The corresponding judgment matrix $\mathrm{K}$ is constructed by comparing matrix A.

$$
\text { Among: } a_{i j}=\left\{\begin{array}{c}
r_{i}-r_{j}, r_{i}>r_{j} \\
1, r_{i}=r_{j} \\
\left(r_{i}-r_{j}\right)^{-1}, r_{i}<r_{j}
\end{array}\right.
$$

In the formula, $r_{i}, r_{j}$ is the rows of the matrix. 
3) Optimal judgment matrix. The matrix $A$ is calculated and the matrix $B$ is optimized. $b_{i j}=\sqrt[n]{\frac{\prod_{l=1}^{n} a_{i l}}{\prod_{l=1}^{n} a_{j l}}}$

Calculation index weight.First of all, the weight of each index ${ }_{w}$ 'is calculated according to the single matrix B value;then, the weight of the $\mathrm{i}$ index of the corresponding value for the normalized, the standard weight of the I index value corresponding to the $w_{i}^{\prime}$. The standard weight $w_{i}$ of the i index value corresponding to the .

$$
w_{i}^{\prime}=\sqrt[n]{\prod_{j=1}^{n} b_{i j}}, i=1,2, \mathrm{~L}, n \quad \text { (4) } \quad w_{i}=\frac{w_{i}^{\prime}}{\sum_{i=1}^{n} w_{i}^{\prime}}, i=1,2, \mathrm{~L}, n
$$

In the above formula, $w_{i}^{\prime}$ is the weight of the ith index, and $w_{i}$ is the normalized weight of the ith index, and $\mathrm{n}$ is the total number of evaluation indexes.

\section{Objective weight is determined by entropy weight method}

The entropy value reflects the amount of useful information provided by each evaluation factor to the decision evaluation. If the entropy of a factor is smaller, the more information is provided by this factor, the greater the weight. Entropy weight method is used to calculate the weight of each index $C_{i j}$ in the evaluation index system of metro operation safety, and the specific calculation process reference [6].

According to this model, the objective weight of each factor index of metro operation safety evaluation index system can be obtained.

\section{calculate the weight of the combination}

On the basis of improving analytic hierarchy process and entropy weight method, a new method of combinatorial empowerment is constructed by introducing game theory. Its purpose is to find consistent between the subjective and objective weights or compromise, make comprehensive weight can not only with the subjective and objective weight deviation minimization, as much as possible and keep the information contained in subjective and objective weights. Specific methods are as follows [7]:

The weight vector $W_{i}^{T}$, which is obtained by different weighting methods, is given by $\mathrm{m}(\mathrm{m}=2)$, and its arbitrary linear combination is: $W=\sum_{i=1}^{m} \alpha_{i} W_{i}^{T}, \alpha_{i}>0$

Therefore, finding the most satisfactory weight vector can be attributed to the optimization of $\mathrm{m}$ linear combination coefficient a in equation (7). The optimization goal is to minimize the dispersion of W with each $W_{i}^{T}$. Therefore, the following countermeasures model is derived:

$$
\min \left\|\sum_{i=1}^{m} \alpha_{i} W_{i}^{T}-W_{j}^{T}\right\|_{2}, j=1,2, \mathrm{~L}, m
$$

The game model is a set of contains a cross planning model of multiple objective function, solve the model is able to get a and a variety of weight assignment method in the overall information coordinated, balanced and consistent comprehensive weighting results.

According to the differential property of the matrix, the first derivative of the formula (6) is:

$\sum_{i=1}^{m} \alpha_{i} W_{j} W_{i}^{T}=W_{j} W_{j}^{T}$

The $\alpha_{i}$ is obtained by Matlab and normalized to $\alpha_{i}^{*}=\alpha_{i} / \sum_{i=1}^{m} \alpha_{i}$

Finally, $\alpha_{i}^{*}$ substituted into equation (6) can be used to find the combined weight vector $W$. 


\section{fuzzy comprehensive evaluation}

In this paper, the fuzzy theory is used to evaluate the safety index system of metro operation, and the specific steps are as follows: first, the evaluation level $V=\{$ excellent, good, general and poor $\}$; And then combined with metro operation by experts to grade indexes at all levels, so as to work out the normalized processing, get the fuzzy evaluation matrix, and according to the results of the above combination empowerment to get the weight of the expert evaluation results are linear weighted; Finally, according to the principle of maximum membership, comprehensive evaluation of subway operation safety is carried out.

\section{Analysis of example}

The north section of Qingdao metro line 3 has been opened since the end of 2015, with a total length of $24.8 \mathrm{~km}$, spanning the southern district, the city and the north district, and li cang-district three administrative regions, with a total of 22 stations, including six transfer stations. Taking Qingdao metro line 3 as an example, this paper evaluates its operation safety.

the subjective weight is calculated by using the improved analytic hierarchy process

First by the panel in three of the importance of the evaluation index scale method are compared, and two for the comparison of $\mathrm{C} 1 \sim \mathrm{C} 4$ corresponding matrix. Indicators of management factors, for example, the comparison matrix of the $\mathrm{K} 3$.

$$
K_{3}=\left[\begin{array}{lllll}
1 & 1 & 2 & 0 & 0 \\
1 & 1 & 2 & 0 & 0 \\
0 & 0 & 1 & 0 & 0 \\
2 & 2 & 2 & 1 & 0 \\
2 & 2 & 2 & 2 & 1
\end{array}\right] A_{3}=\left[\begin{array}{ccccc}
1 & 1 & 3 & 1 / 3 & 1 / 5 \\
1 & 1 & 3 & 1 / 3 & 1 / 5 \\
1 / 3 & 1 / 3 & 1 & 1 / 6 & 1 / 8 \\
3 & 3 & 6 & 1 & 1 / 2 \\
5 & 5 & 8 & 2 & 1
\end{array}\right] \quad B_{3}=\left[\begin{array}{ccccc}
1 & 1 & 2.4395 & 0.3749 & 0.2187 \\
1 & 1 & 2.4395 & 0.3749 & 0.2187 \\
0.4099 & 0.4099 & 1 & 0.1537 & 0.0896 \\
2.6673 & 2.6673 & 6.5068 & 1 & 0.5833 \\
4.5731 & 4.5731 & 11.1560 & 1.7145 & 1
\end{array}\right]
$$

The judgment matrix A3 is obtained by comparison matrix $\mathrm{K} 3$ and formula 5.

The matrix 3 was optimized by formula 6 , and matrix B3 was obtained.

The weights of each indicator can be obtained by formula 7 and formula $8(0.1036,0.1036$, $0.0425,0.2764,0.4739$ ).

In the same way, the weight of other secondary indexes and the weight $w_{0}$ of each index are obtained.

\section{calculation of objective weight by entropy weight method}

Eight experts were randomly selected to form a jury to grade the operating evaluation indexes of Qingdao metro line 3. Indicators of management factors, for example, and set into four levels, excellent $(90,100)$, good $(80,90)$,poor $(70,80)$, difference $(60,70)$, the scores of each secondary index are shown in table 1.

Table 1 quantization value of the index

\begin{tabular}{ccccccccc}
\hline evaluating indicator & 1 & 2 & 3 & 4 & 5 & 6 & 7 & 8 \\
\hline $\mathrm{C}_{31}$ & 85 & 75 & 85 & 90 & 80 & 85 & 80 & 85 \\
$\mathrm{C}_{32}$ & 85 & 80 & 80 & 90 & 85 & 80 & 85 & 80 \\
$\mathrm{C}_{33}$ & 75 & 75 & 80 & 85 & 75 & 85 & 80 & 80 \\
$\mathrm{C}_{34}$ & 85 & 90 & 90 & 85 & 80 & 90 & 85 & 90 \\
$\mathrm{C}_{35}$ & 95 & 90 & 90 & 95 & 85 & 90 & 85 & 90 \\
\hline
\end{tabular}

On the basis of the data in table 1, combined with the formula (1), (2), (3) to calculate the index weights of C31 microcomputer C35 for $(0.0667,0.0433,0.0600,0.0433,0.0600)$, the weights are similarly can calculate the other secondary.

\section{the comprehensive weight is calculated by using combination weighting method}

According to the entropy value method and the improved analytic hierarchy process to calculate the subjective and objective weights and formula (11) to the optimization of comprehensive empowerment theory solutions of first order linear equations, the normalized by subjective weight and objective weight coefficient $=0.4012$, respectively, $=0.5998$, (9) can be combined by the type of weight. 


\section{fuzzy comprehensive evaluation}

In order to make the subway operation safety assessment results as accurate as possible, using questionnaire survey asked a panel of eight experts, to evaluate each of the factors in the secondary indexes, through the survey results are statistics and finishing, management factors, for example, the fuzzy evaluation matrix is zero.

$$
B_{3}=\left[\begin{array}{cccc}
0.44 & 0.38 & 0.16 & 0.02 \\
0.38 & 0.44 & 0.18 & 0 \\
0.36 & 0.32 & 0.3 & 0 \\
0.46 & 0.30 & 0.24 & 0 \\
0.54 & 0.36 & 0.1 & 0
\end{array}\right]
$$

$B_{3} \bullet W_{3}=(0.1113 ; 0.0917 ; 0.0498 ; 0.0012)$

The other criterion layer fuzzy evaluation vector is obtained. By the fuzzy evaluation matrix D, the weight vector and the fuzzy evaluation matrix D are obtained by the fuzzy evaluation matrix D.

$$
w_{0} \bullet D=\left[\begin{array}{llll}
0.3937 & 0.3937 & 0.1374 & 0.0752
\end{array}\right] \bullet\left[\begin{array}{llll}
0.0826 & 0.0839 & 0.0259 & 0.0014 \\
0.2009 & 0.1844 & 0.0672 & 0.0032 \\
0.1113 & 0.0917 & 0.0498 & 0.0012 \\
0.0402 & 0.0397 & 0.0156 & 0.0011
\end{array}\right]=\left[\begin{array}{llll}
0.1299 & 0.1212 & 0.0447 & 0.0021
\end{array}\right]
$$

According to the principle of maximum membership, the operation safety level of Qingdao metro line 3 is excellent.

\section{Conclusion}

(1) The traditional analytic hierarchy process 9 scaling method, the evaluation index to compare the scale division is more, it leads to several experts in the index to judge the existence difference and partial probability, led to the evaluation index weight is not accurate. However, the improved analytic hierarchy process adopts the three scale, which is only $0,1,2$ and 3 choices when comparing the two indexes. The choice is more simple and reliable.

(2) The introduction of game theory, balance and coordination of the improved analytic hierarchy process and entropy value method, the role and impact of maximum was overcome by a single subjective or objective weight respectively, make evaluation result more scientific and reasonable.

(3) Numerical example results show that the Qingdao metro line 3 security level for optimal, and passenger flow factor, equipment factor, management factor, environmental factors, the corresponding security level is good, excellent, excellent, respectively. Therefore, in order to improve the safety level of metro operation, the passenger flow factor should be considered, and passenger guidance should be conducted.

\section{References:}

[1] JIA.S.K, WEN.X.H, LIN. D.J,JIANG.Z.R. Research on Safety Evaluation technology of metro operation system based on analytic hierarchy process $[\mathrm{J}]$. Journal of Chinese security science, 2008,(05):137-141.

[2] JIANG.T, ZHOU.H.W. Risk assessment of metro operating system based on entropy method [J]. China water transportation (second half), 2015,15(07):53-56.

[3] WANG.Y.H, HUANG.Y.K, LI.M. Safety evaluation of urban rail line operation based on combination empowerment method [J/OL]. Journal of Tongji university (natural science edition), 2013,41(08):1243-1248.

[4] TANG.J.J,WANG.P,LUO.X.P. Design of the information system for the management of hazardous source in subway system $[\mathrm{J} / \mathrm{OL}]$. Journal of civil engineering and management, 2016,33(04):63-67+82. 
[5] CHANG.J,ZHANG.Z.J,WANG.J.L. Application of three-scale fuzzy analytic hierarchy process in bridge safety assessment [J]. China safety production science and technology, 2014,10(08):156-161.

[6] LI.L,ZHAO.J.X,LI.F.Evaluation on management performance of metro engineering projects based on entropy and grey theory $[\mathrm{J}]$. Journal of Qingdao polytechnic university, 2014,(03):50-55+60. 